\title{
EXAMPLES FOR THE INFINITE DIMENSIONAL MORSE LEMMA*
}

\author{
MICHAEL BUCHNER ${ }^{\dagger}$, JERROLD MARSDEN $^{\ddagger}$ AND STEPHEN SCHECTER ${ }^{\S}$
}

\begin{abstract}
Examples are presented which show how to use the Morse lemma in specific infinite dimensional examples and what can go wrong if various hypotheses are dropped. One of the examples shows that the version of the Morse lemma using singularity theory can hold, yet the hypotheses of the Morse-Palais and Morse-Tromba lemmas fail. Another example shows how to obtain a concrete normal form in infinite dimensions using the splitting lemma and hypotheses related to those in the Morse-Tromba lemma. An example of Dancer is given which shows that for the validity of the Morse lemma in Hilbert space, some hypotheses on the higher order terms must be made in addition to smoothness, if the quadratic term is only weakly nondegenerate. A general conjecture along these lines is made.
\end{abstract}

Introduction. In this paper we discuss several examples relevant to the Morse lemma and singularity theory in infinite dimensions.

We begin with some historical comments on the various methods that have been used to prove the Morse lemma. The original method of Morse uses induction on the dimension of the space and does not, as given, apply to infinite dimensions. See Milnor [1963] for this proof. The Palais method was introduced in Palais [1963]. It is a modification of the original method that works in Hilbert space under the hypothesis of strong nondegeneracy of the quadratic term.

The Moser-Weinstein method is a variant of the singularity theory method described in Golubitsky and Marsden [1983] (this issue, pp. 1037-1044). It was adapted to the Morse lemma by Palais [1969]. Rather than directly join the quadratic part $f$ to $f+p$ by $f+t p$, as in the preceding paper, one joins $d f$ to $d f+d p$ by $d f+t d p$. Palais' [1969] theorem states the following: if $E$ is a Banach space, $h: E \rightarrow \mathbb{R}$ is $C^{3}, D h(0)=0$, and $D^{2} h(0)$, regarded as a map of $E$ to $E^{*}$, is an isomorphism, then there is a $C^{1}$ diffeomorphism $\phi$ defined on a neighborhood of 0 in $E$ such that

and

$$
\phi(0)=0, \quad D \phi(0)=I(=\text { identity }),
$$

$$
h(\phi(x))=h(0)+\frac{1}{2} D^{2} h(0) \cdot(x, x) \text {. }
$$

In Hilbert space this result reduces to that in Palais [1963]. We call the condition on $D^{2} h(0)$ strong nondegeneracy. If the map of $E$ to $E^{*}$ associated to $D^{2} h(0)$ is injective, we say $D^{2} h(0)$ is weakly nondegenerate.

The Morse-Tromba lemma was introduced in Tromba [1976]. It is motivated by the fact that in many elliptic variational problems one does not have strong nondegeneracy of the quadratic term. Rather, this is changed to weak nondegeneracy at the expense of putting special hypotheses on the nonlinear terms. The necessity of weak nondegeneracy occurred already for Hamiltonian systems in Marsden [1968]. Tromba's original proof was an adaptation of Palais' [1963] proof. A proof of the Morse-Tromba lemma using the Moser-Weinstein method was given in Choquet-Bruhat, Fischer and Marsden [1979]. The Morse-Tromba lemma is Theorem B of Golubitsky and Marsden [1983]. The singularity theory method, described in that paper, yields a result strictly stronger than Tromba's. Examples 5 and 6 below illustrate this.

\footnotetext{
*Received by the editors June 28, 1982, and in revised form October 29, 1982.

${ }^{\dagger}$ Department of Mathematics, University of Maryland, College Park, Maryland 20742.

${ }^{\ddagger}$ Department of Mathematics, University of California, Berkeley, California 94720.

${ }^{\S}$ Department of Mathematics, North Carolina State University, Raleigh, North Carolina 27650.
} 
For spaces admitting a duality map (such as Hilbert space or $W^{s, p}$ spaces with $p$ even), the Morse-Tromba lemma is valid for $C^{2}$ functions with $C^{1}$ changes of coordinates (by Remark (e) following Theorem B of Golubitsky and Marsden [1983]). We do not know a $C^{2}$ counterexample if $E$ is a general Banach space. We conjecture that there is not such an example.

The Morse-Tromba lemma suggests the question: can the Morse-Palais lemma be generalized without putting conditions on the higher order terms? We conjecture that the answer is no. More precisely,

CONJECTURE. Let $E$ be a Banach space and let $B: E \times E \rightarrow \mathbb{R}$ be a continuous symmetric bilinear map such that $x \mapsto B(x, \cdot)$ is not an isomorphism of $E$ and $E^{*}$. Let $f(x)=\frac{1}{2} B(x, x)$. Then there is a $C^{3}$ map $p: E \rightarrow \mathbb{R}$ with $p(0)=0, D p(0)=0$, and $D^{2} p(0)=0$ such that $f$ and $f+p$ are not $C^{1}$ right equivalent.

For $E$ a Hilbert space, this conjecture has been verified by E. N. Dancer (private communication). His class of examples is presented below in Example 8 .

In the examples that follow, the labels $(E 1),(E 2),(T 1),(T 2),(S 1),(S 2)$, Theorem $A$ and Theorem B refer to Golubitsky and Marsden [1983]. A couple of these examples are simple and well known but are included for completeness.

Example 1. This example shows that nondegeneracy of $D^{2} h(0)$ in the sense of $(T 1)$ is not sufficient for the validity of the Morse lemma. Let $E=l_{2}$ and let $h$ be the $C^{\infty}$ function

$$
h(x)=\frac{1}{2} \sum_{n=1}^{\infty} \frac{1}{n} x_{n}^{2}-\frac{1}{3} \sum_{n=1}^{\infty} x_{n}^{3} .
$$

Let $\langle x, y\rangle=\sum_{n=1}^{\infty}(1 / n) x_{n} y_{n}$. Then (T1) holds with $T=I$. However (T2) fails, since the only possibility would be

$$
\nabla h(x)_{n}=x_{n}-n x_{n}^{2}, \quad n=1,2, \cdots
$$

which is not defined on open sets in $l_{2}$. Indeed, the Morse lemma fails for this function. The quadratic term has no zeros other than the origin, yet $h$ vanishes on the sequence $(0,0, \cdots, 3 / 2 n, 0, \cdots)$, which approaches 0 in $l_{2}$. If the cubic term is changed to $\frac{1}{3} \sum_{n=1}^{\infty}(1 / n) x_{n}^{3}$ then the gradient exists and the Morse-Tromba lemma applies.

Example 2. This example shows that Tromba's hypotheses (T1) and (T2), but not those of the Morse-Palais lemma, can be expected to hold for many elliptic variational problems. If $\Omega$ is a bounded region in $\mathbb{R}^{n}$ with smooth boundary, $W^{s, p}\left(\Omega, \mathbb{R}^{m}\right)$ denotes the Sobolev space of maps $u: \Omega \rightarrow \mathbb{R}^{m}$ whose derivatives up to order $s$ are in $L^{p}$ (see Friedman [1969], for example). For $p=2$ we write $W^{s, 2}=H^{s}$. If $m=1$ we write $W^{s, p}(\Omega, \mathbb{R})=W^{s, p}(\Omega)$.

Let us begin with the one-dimensional case.

(a) Let $E=H^{1}([a, b])$. We define the function $g: E \rightarrow \mathbb{R}$ by

$$
g(u)=\int_{a}^{b}[u(x)]^{2} d x+\int_{a}^{b}[u(x)]^{3} d x=f(u)+p(u) .
$$

Composition properties of Sobolev spaces (Palais [1968]) show that $g$ is $C^{\infty}$. Considered as a linear map $E \rightarrow E^{*}$, the bilinear map $D^{2} g(0)$ is $u \mapsto\left(v \mapsto 2 \int_{a}^{b} u v\right)$. This map is injective but not surjective. For example the delta function $\delta_{x}(v)=v(x)$ for $a<x<b$ is in $E^{*}$ but not in the image of $D^{2} g(0): E \rightarrow E^{*}$. Thus the hypotheses of the Morse-Palais lemma do not hold. (If $\int_{a}^{b}[u(x)]^{2} d x$ is replaced by $\int_{a}^{b}[u(x)]^{2} d x+\int_{a}^{b}\left[u^{\prime}(x)\right]^{2} d x$, then 
the hypotheses of the Morse-Palais lemma do hold; this quadratic functional is similar to the functionals used in the variational approach to geodesics.)

On the other hand, let $\langle\cdot, \cdot\rangle$ be the $L^{2}$ inner product on $H^{1}$. Then the gradient $\nabla g(u)$ relative to $\langle\cdot, \cdot\rangle$ is given by $\nabla g(u)=2 u+3 u^{2}$, which is $C^{\infty}$. Moreover, $D \nabla g(0)$ $=2 I$. Consequently, Tromba's Morse lemma applies, so $g$ can be transformed to the functional $\int_{a}^{b}[u(x)]^{2} d x$.

In this example the transformation can be seen directly. Observe that $g(u)$ can be written as $g(u)=\int_{a}^{b}\left[u(x)(1+u(x))^{1 / 2}\right]^{2} d x$. Now if $\phi:(c, d) \subset \mathbb{R} \rightarrow \mathbb{R}$ is $C^{\infty}$ then $u \mapsto$ $\phi \circ u$ is $C^{\infty}$ on $\left\{u \in H^{1} \mid c<u(x)<d\right.$ for all $\left.x \in[a, b]\right\}$. Hence the map $u \mapsto u(1+u)^{1 / 2}$ is $C^{\infty}$ on $\left\{u \in H^{1} \mid-1<u(x)<\infty\right.$ for all $\left.x \in[a, b]\right\}$, has derivative the identity at $u=0$, and hence is a local diffeomorphism.

Tromba's proof of his Morse lemma applied to this example also yields the transformation $u \mapsto u(1+u)^{1 / 2}$. So does the proof of Theorem A. For if one solves $p=-d f \cdot A$ by $A(u)=-u^{2} / 2$ and $d p=d f \circ R$ by $R(u) \cdot v=3 u v / 2$, one obtains $A(u)=$ $-u^{2} / 2$ and for $A_{t}(u)$ we get the expression $-[1+3 t u / 2]^{-1} u^{2} / 2$. Note that $A(u)=$ $-\int_{0}^{1} \tau R(\tau u) \cdot u d \tau$, in agreement with Remark (b) following Theorem A of Golubitsky and Marsden [1983]. Integrating this vector field leads to the inverse of the transformation $u \mapsto u(1+u)^{1 / 2}$.

(b) We now sketch a typical multiple integral variational problem in higher dimensions. (Proofs rely on standard elliptic theory and Sobolev estimates, which are omitted here.) Let $E$ be $W_{\partial}^{s, p}(\Omega)$, the $W^{s, p}$ functions which are zero on $\partial \Omega$, and let $s>n / p+1$. Consider $h: E \rightarrow \mathbb{R}$ defined by

$$
h(u)=\int_{\Omega} W(D u) d x+\int_{\Omega} K(u) d x
$$

where $W$ is a smooth function of $\mathbb{R}^{n}$ to $\mathbb{R}, K$ is a smooth function of $\mathbb{R}$ to $\mathbb{R}$, and $D u(x)$ is identified with a column vector or a point in $\mathbb{R}^{n}$. Suppose that

$$
W(0)=0, \quad D W(0)=0, \quad K(0)=D K(0)=D^{2} K(0)=0
$$

and

$$
D^{2} W(0) \cdot(\xi, \eta) \geq c\|\xi\|\|\eta\| \quad \text { for all } \xi, \eta \in \mathbb{R}^{n}, \quad \text { where } c>0 .
$$

Standard Sobolev inequalities (cf. Palais [1968, Thm. 9.10]) show that $h$ is a smooth function. Let $\langle\cdot, \cdot\rangle$ on $E$ be given by

$$
\langle u, v\rangle=\int_{\Omega} D u \cdot D v d x
$$

Then

$$
D h(u) \cdot v=\int_{\Omega} D W(D u) \cdot D v d x+\int_{\Omega} D K(u) \cdot v d x
$$

and

$$
D^{2} h(0) \cdot(u, v)=\int_{\Omega}(D u)^{T} M(D v) d x
$$

where $D^{2} W(0) \cdot(\xi, \eta)=\xi^{T} M \eta$ for an $n \times n$ positive definite matrix $M$. Then $(T 1)$ holds for $(T u)(x)=\Delta^{-1} \operatorname{div}(M D v)$, using the classical fact that $\Delta: W_{\partial}^{s, p}(\Omega) \rightarrow W^{s-2, p}(\Omega)$ is an 
isomorphism (Friedman [1969]). Also, $T$ is an isomorphism on these spaces, for, as is readily checked, $\operatorname{div}(M D u)$ is elliptic with trivial kernel. (T2) holds with

$$
\nabla h(u)=\Delta^{-1} \operatorname{div}[D W(D u)]-\Delta^{-1} D K(u) .
$$

For this example, again the hypotheses of the Morse-Tromba lemma hold, but those of the Morse-Palais lemma do not. Examples like this occur in minimal surfaces (see Tromba [1981]) and in elasticity (see Chillingworth, Marsden and Wan [1982] and Marsden and Hughes [1983]).

Example 3. (a) This example will show that Theorem A is not limited to functions of the form quadratic + higher order (as the Morse-Tromba lemma is). Let $E=H^{1}([a, b])$ and let

$$
g(u)=\int_{a}^{b}[u(x)]^{3} d x+\int_{a}^{b}[u(x)]^{4} d x
$$

Let

$$
f(u)=\int_{a}^{b}[u(x)]^{3} d x \text { and } p(u)=\int_{a}^{b}[u(x)]^{4} d x .
$$

The equation $p(u)=-D f(u) \cdot A(u)$ can be solved by $A(u)=-u^{2} / 3$, and $D p(u)=$ $D f(u) \circ R(u)$ is solved by $R(u) \cdot v=4 u v / 3$. Note that

$$
A(u)=-\int_{0}^{1} \tau^{2} R(\tau u) \cdot u d \tau .
$$

(See Remark (b) following Theorem A in Golubitsky and Marsden [1983].) Hence $g$ can be transformed to $f$ by a $C^{\infty}$ transformation. This transformation, as in Example 2a, can be found directly by writing $g(u)=\int_{a}^{b}\left[u(x)(1+u(x))^{1 / 3}\right]^{3} d x$. Then $\phi(u)=u(1+$ $u)^{1 / 3}$ is a suitable transformation.

An easy calculation shows that the diffeomorphism obtained by integrating the vector field $A_{t}(u)=-[1+4 t u / 3]^{-1} u^{2} / 3$ is the inverse of $\phi(u)=u(1+u)^{1 / 3}$.

(b) Let $E=H^{1}([a, b])$ and let $g(u)=f(u)+p(u)$ where $f(u)=\int_{a}^{b}[u(x)]^{3} d x$ and $p(u)=\left\{\int_{a}^{b}[u(x)]^{2} d x\right\}^{2}$. Theorem $\mathrm{A}$ applies to $g$ and shows that $g$ can be transformed to $f$. Indeed, $p(u)=-D f(u) \cdot A(u)$ can be solved by $A(u)=-\frac{1}{3} \int_{a}^{b}[u(x)]^{2} d x$ and $D p(u)=$ $D f(u) \circ R(u)$ is solved by $R(u) \cdot v=\frac{4}{3} \int_{a}^{b} u(x) v(x) d x$ (both $A(u)$ and $R(u) \cdot v$ are constant functions). Note that $A(u)=\int_{0}^{1} \tau R(\tau u) \cdot u d \tau$. On the other hand there does not seem to be any explicit diffeomorphism that one could write down by inspection of $g$. The conjugating diffeomorphism is given by integrating

$$
\frac{\partial \phi}{\partial t}(u, t)=\frac{-\frac{1}{3} \int_{a}^{b}[\phi(u, t)]^{2} d x}{1+\frac{4}{3} t \int_{a}^{b} \phi(u, t) d x}, \quad \phi(u, 0)=u
$$

and setting $t=1$. It seems unlikely this could be solved explicitly in any simple fashion.

Example 4. We now give an example of a function $h$ which is $C^{3},(T 1)$ holds, $\nabla h$ exists and is continuous but is not $C^{1}$, and yet the Morse lemma fails. lemma.

Thus the hypothesis that $\nabla h$ be $C^{1}$ cannot be weakened to $C^{0}$ in the Morse-Tromba

Let $E=L^{q}([0,1])$ and let $\phi: \mathbb{R} \rightarrow \mathbb{R}$ be a $C^{\infty}$ function such that $\phi^{\prime}(\lambda)=1,-1 \leq \lambda \leq 1$ and $\phi^{\prime}(\lambda)=0$ if $|\lambda| \geq 2$. We assume $\phi$ is monotone increasing with $\phi=-M$ for $\lambda \leq-2$ 
and $\phi=M$ for $\lambda \geq 2$. Let $h: E \rightarrow \mathbb{R}$ be given by

$$
h(u)=\frac{1}{2} \int_{0}^{1}[u(x)]^{2} d x+\frac{1}{3} \int_{0}^{1} \phi\left([u(x)]^{3}\right) d x=f(u)+p(u) .
$$

For $q \geq 2, f$ is clearly $C^{\infty}$. Let $\langle\cdot, \cdot\rangle$ be the $L^{2}$ inner product on $L^{q}([0,1])$; then $(T 1)$ holds with $T=I$. We claim that if $q$ is an integer, then $p$ is $C^{q-1}$ but not $C^{q}$, and $\nabla p$ exists, is continuous, but is not $C^{1}$. Thus with $q \geq 4$ we get a $C^{3}$ function. Let us indicate the proof of these facts for $q=4$.

To prove that $p$ is $C^{3}$, we let $\psi(\lambda)=\phi\left(\lambda^{3}\right)$. By Taylor's theorem,

$$
\psi(\lambda)=\sum_{k=0}^{3} \psi^{(k)}\left(\lambda_{0}\right) \frac{\left(\lambda-\lambda_{0}\right)^{k}}{k !}+R\left(\lambda, \lambda_{0}\right)\left(\lambda-\lambda_{0}\right)^{3}
$$

where $\lim _{\lambda \rightarrow \lambda_{0}} R\left(\lambda, \lambda_{0}\right)=0$ and, from the definition of $\phi, \psi^{(k)}$ and $R$ are bounded smooth functions. Thus, suppressing the argument $x$, for $u$ and $u_{0}$ continuous functions of $x$ we have the identity

$$
3 p(u)=\sum_{k=0}^{3} \int_{0}^{1} \psi^{(k)}\left(u_{0}\right) \frac{\left(u-u_{0}\right)^{k}}{k !} d x+\int_{0}^{1} R\left(u, u_{0}\right)\left(u-u_{0}\right)^{3} d x
$$

Since $\psi^{(k)}\left(\lambda_{0}\right)$ and $R\left(\lambda, \lambda_{0}\right)$ are bounded continuous, $\psi^{(k)}\left(u_{0}\right)$ (resp. $\left.R\left(u, u_{0}\right)\right)$ extends to a continuous mapping from $L^{4}$ (resp. $L^{4} \times L^{4}$ ) to $L^{4}$. Using this fact and the Schwarz inequality, it follows that $p(u)$ depends continuously on $u \in L^{4}$, and each integral above depends continuously on $\left(u, u_{0}\right) \in L^{4} \times L^{4}$. Thus the identity holds for all $\left(u, u_{0}\right) \in L^{4} \times$ $L^{4}$. Since $\psi^{(k)}\left(u_{0}\right)(k=0,1,2,3)$ is bounded, $\left(v_{1}, \cdots, v_{k}\right) \mapsto \int_{0}^{1} \psi^{(k)}\left(u_{0}\right) v_{1} \cdots v_{k} d x$ is a bounded multilinear functional on $L^{4}$. Using the Schwarz inequality and the Lebesgue dominated convergence theorem we see that the mapping that associates to $u_{0} \in L^{4}$ the $k$-multilinear functional $\left(v_{1}, \cdots, v_{k}\right) \mapsto \int_{0}^{1} \psi^{(k)}\left(u_{0}\right) v_{1} \cdots v_{k} d x$ is continuous from $L^{4}$ to the bounded multilinear functionals on $L^{4}$. Also, $\lim _{u \rightarrow u_{0}} R\left(u, u_{0}\right)=0$. It follows from the converse to Taylor's theorem (Abraham and Robbin [1967]) that $p$ is $C^{3}$.

Now an easy check shows that $\nabla p(u)$ exists and is given by

$$
\nabla p(u)=\frac{\psi^{\prime}(u)}{3}
$$

If this were $C^{1}$, its derivative would be

$$
u \mapsto\left(v \rightarrow \frac{\psi^{\prime \prime}(u) v}{3} \equiv P(u) \cdot v\right) .
$$

Choose a number $a$ such that $\psi^{\prime \prime}(a) / 3 \neq 0$ and let

and

$$
u_{n}= \begin{cases}a & \text { on }[0,1 / n] \\ 0 & \text { elsewhere }\end{cases}
$$

$$
v_{n}= \begin{cases}\sqrt[4]{n} & \text { on }[0,1 / n] \\ 0 & \text { elsewhere }\end{cases}
$$

Then one sees that $u_{n} \rightarrow 0$ in $L^{4},\left\|v_{n}\right\|=1$ in $L^{4}$, but $P\left(u_{n}\right) \cdot v_{n} \nrightarrow 0$ in $L^{4}$. Since $P(0)=0$, $\nabla p(u)$ is not $C^{1}$. (One sees in a similar way that $p$ is not $C^{4}$.) 

namely

Finally, we note that $h$ has a sequence of critical points $u_{n}$ approaching the origin,

$$
u_{n}= \begin{cases}-1 & \text { on }[0,1 / n] \\ 0 & \text { on }(1 / n, 1]\end{cases}
$$

Since this is not true for $f$, the Morse lemma cannot hold for $h$.

Example 5. We give an example to show that $(E 1),(E 2)$ and $(T 1)$ can hold, without (T2) holding. Thus, the Morse lemma is valid, Theorem A applies, but Tromba's Theorem $\mathrm{B}$ does not.

Let $E=l_{1}$, the space of sequences $x_{n}$ with $\sum_{n=1}^{\infty}\left|x_{n}\right|<\infty$. Let $h=f+p$ where

$$
f(x)=\frac{1}{2} \sum_{n=1}^{\infty} x_{n}^{2}=\frac{1}{2}\langle x, x\rangle
$$

$\langle\cdot, \cdot\rangle$ being the usual $l_{2}$ inner product, and

$$
p(x)=\left(\sum_{n=1}^{\infty} x_{n}\right) x_{1}^{2}+x_{2}^{3}+x_{3}^{3}+\cdots .
$$

Since $p$ is induced by a continuous trilinear map, $h$ is $C^{\infty}$. Also, $(T 1)$ holds with $T=I$. (E1) holds with

$$
A(x)=-\left(\left(\sum_{n=1}^{\infty} x_{n}\right) x_{1}, x_{2}, x_{3}, \cdots\right)
$$

and (E2) holds with

$$
R(y) \cdot u=\left(\left(\sum_{i=1}^{\infty} u_{i}\right) y_{1}+2\left(\sum_{i=1}^{\infty} y_{i}\right) u_{1}, 3 u_{2} y_{2}, 3 u_{3} y_{3}, \cdots\right)
$$

as is easily checked. However (T2) cannot hold using the $l_{2}$ inner product (or, by Remark (c), following Theorem B, any inner product such that (T1) holds). If $\nabla h$ exists, so does $\nabla p($ since $\nabla f(x)=x)$. But $\nabla p$ would be

$$
\nabla p(x)=\left(x_{1}^{2}+2 x_{1}\left(\sum_{n=1}^{\infty} x_{n}\right), 3 x_{2}^{2}+x_{1}^{2}, 3 x_{3}^{2}+x_{1}^{2}, \cdots\right)
$$

which is not in $l_{1}$. Note also that $R(y)$ does not have an everywhere defined $l_{2}$ adjoint; see Remark (b) following Theorem $B$.

Example 6. A variation on Examples 2 and 5 gives an example which is a prototype for problems in elasticity in which two bodies are in contact at a point. Like Example 5, this example has (E1), (E2) and (T1) holding, but not (T2).

Let $\Omega \subset \mathbb{R}^{n}$ be a region with smooth boundary and $0 \in \Omega$; for instance, let $\Omega$ be the unit disk in the plane. Let $E=W_{\partial}^{s, p}, s>n / p+1$, the Sobolev space $W^{s, p}$ with Dirichlet boundary conditions, and let $h: E \rightarrow \mathbb{R}$ be given by

$$
h(u)=\frac{1}{2} \int_{\Omega}\|D u\|^{2} d x+u(0) \int_{\Omega} u^{2} d x .
$$


As above, $h$ is $C^{\infty}$. Let $\langle u, v\rangle$ on $E$ be defined by $\langle u, v\rangle=\int_{\Omega}(D u \cdot D v) d x$. Then $(T 1)$ holds with $T=I$. Let

$$
f(u)=\frac{1}{2} \int_{\Omega}\|D u\|^{2} d x \text { and } p(u)=u(0) \int_{\Omega} u^{2} d x .
$$

We show that $p$ cannot have a gradient $\nabla p$ with respect to $\langle\cdot, \cdot\rangle$ (except in the case $s=1, n=1$ ), and thus (T2) cannot hold (except in the case $s=1, n=1$, in which case (T2) holds) for $\nabla p$ would have to satisfy

$$
\int_{\Omega} D v \cdot D(\nabla p(u)) d x=\int_{\Omega} D v \cdot D u d x+v(0) \int_{\Omega} u^{2} d x+2 u(0) \int_{\Omega} u v d x .
$$

This implies

$$
\Delta(u-\nabla p(u))=\left(\int_{\Omega} u^{2}\right) \delta_{0}+2 u(0) u \quad \text { (as distributions) }
$$

where $\delta_{0}$ is the Dirac delta function at the origin. But $\delta_{0}$ is not in $W^{s-2, p}$ unless $s=1$ and $n=1$, in which case $\delta_{0} \in W^{-1, p}$. In this latter case $\nabla p(u)$ is given by the formula $u-\left(\int_{\Omega} u^{2}\right) \Delta^{-1} \delta_{0}-2 u(0) \Delta^{-1} u$ (using the fact that $\Delta: W_{\partial}^{1, p} \rightarrow W^{-1, p}$ is an isomorphism). Thus Tromba's hypotheses are satisfied only in the case $s=1, n=1$. In the case $s=1$, $n=1, p=2$, the Palais-Morse lemma hypotheses are also satisfied since $\langle\cdot, \cdot\rangle$ is the Hilbert space inner product for $H_{\partial}^{1}$.

On the other hand, for arbitrary $s$ and $n$ (with $s>n / 2+1$ ), $p=-d f \cdot A$ is solved by $A(u)=u(0) \Delta^{-1} u$, and $d p=d f \circ R$ is solved by $R(u) \cdot v=-2 u(0) \Delta^{-1} v-v(0) \Delta^{-1} u$ (note that $\left.A(u)=-\int_{0}^{1} \tau R(\tau u) \cdot u d \tau\right)$, so Theorem A applies.

Example 7 below concerns the splitting lemma under hypotheses compatible with Tromba's Morse lemma. We shall use the splitting lemma from the previous paper for problems in which there is an additional parameter.

Example 7. As in Example 2, let $s>n / p+1$ and $E=W_{\partial}^{s, p}(\Omega)$, the $W^{s, p}$ space with Dirichlet boundary conditions. Assume that $\lambda_{0}$ is a simple eigenvalue of the Laplacian $\Delta$ on $\Omega$ and define $h: E \times \mathbb{R} \rightarrow \mathbb{R}$ by

$$
h(u, \lambda)=\int_{\Omega}\left[\frac{1}{2}\|D u\|^{2}+\frac{1}{2}\left(\lambda_{0}+\lambda\right) u^{2}+G(u)\right] d x,
$$

where $G(t)=t^{3}+$ (higher order terms) is a $C^{\infty}$ function from $\mathbb{R}$ to $\mathbb{R}$. We shall apply the splitting lemma to $h$ and bring it to normal form. We find that

(a) $D h(u, \lambda) \cdot(v, \mu)=\int_{\Omega}\left[D u \cdot D v+\left(\lambda_{0}+\lambda\right) u v+\frac{1}{2} \mu u^{2}+G^{\prime}(u) v\right] d x$,

(b) $D^{2} h(u, \lambda) \cdot((v, \mu), \quad(w, v))=\int_{\Omega}\left[D v \cdot D w+\left(\lambda_{0}+\lambda\right) v w+G^{\prime \prime}(u) v w+\nu u v+\right.$ $\mu u w] d x$,

(c) $D^{3} h(u, \lambda) \cdot((v, \mu),(w, \nu),(y, \sigma))=\int_{\Omega}\left[G^{\prime \prime \prime}(u) v w y+\sigma v w+\nu y v+\mu y w\right] d x$.

Define $\langle v, w\rangle=\int_{\Omega} D v \cdot D w d x$. Then $D^{2} h(0,0) \cdot((v, \mu), \quad(w, v))=\int_{\Omega}[D v \cdot D w+$ $\left.\lambda_{0} v w\right] d x=\langle T v, w\rangle$ where $T v=\left(I-\lambda_{0} \Delta^{-1}\right) v$. Since $\Delta$ is elliptic, $T$ is Fredholm of index 0 . The null space of $T$ is $N(T)=\left\langle u_{0}\right\rangle$, where $u_{0}$ is an eigenfunction of $\Delta$ for the eigenvalue $\lambda_{0}$. The range of $T$ is $R(T)=\left\langle u_{0}\right\rangle^{\perp}$, the space of vectors in $E$ that are $L^{2}$-orthogonal to $u_{0}$. Let $P$ be projection onto $\left\langle u_{0}\right\rangle^{\perp}$. Write $u \in E$ as $u=\alpha u_{0}+\tilde{u}$, $\tilde{u} \in\left\langle u_{0}\right\rangle^{\perp}$.

Let $\nabla h(u, \lambda)=\left(I-\left(\lambda_{0}+\lambda\right) \Delta^{-1}\right) u-\Delta^{-1} G^{\prime}(u)$. Then $D h(u, \lambda) \cdot(v, 0)=$ $\langle\nabla h(u, \lambda), v\rangle$, and $P \nabla h$ is what was called $\nabla_{y} h$ in the splitting lemma. Solving $P \nabla h(u, \lambda)=0$ using the implicit function theorem gives a function $\tilde{u}(\alpha, \lambda)$ such that 
$P \nabla h\left(\alpha u_{0}+\tilde{u}(\alpha, \lambda), \lambda\right)=0$. Now $\tilde{u}(0,0)=0$, and $D \tilde{u}(0,0)=0$ because the kernel of $P \circ D \nabla h(0,0)$ is $\left\langle u_{0}\right\rangle \times \mathbb{R}$. Clearly $\tilde{u}(0, \lambda)=0$ for all $\lambda$, since $\nabla h(0, \lambda)=0$ for all $\lambda$.

Let $v$ denote a typical element of $\left\langle u_{0}\right\rangle^{\perp}$. Let $k(\alpha, v, \lambda)=h\left(\alpha u_{0}+\tilde{u}(\alpha, \lambda)+v, \lambda\right)$, so that $D k(\alpha, 0, \lambda) \cdot(0, w, 0)=0$ for all $w \in\left\langle u_{0}\right\rangle^{\perp}$. There is then an $(\alpha, \lambda)$-dependent change of coordinates $v=\eta_{(\alpha, \lambda)}(\bar{v})$ with $\eta_{(\alpha, \lambda)}(0)=0$ and $D \eta_{(\alpha, \lambda)}(0)=I$, such that

$$
k\left(\alpha, \eta_{(\alpha, \lambda)}(\bar{v}), \lambda\right)=k(\alpha, 0, \lambda)+\frac{1}{2} D^{2} k(\alpha, 0, \lambda)(\bar{v}, \bar{v}) .
$$

To find a normal form for $k$ (and hence $h$ ) it remains to find a normal form for $g(\alpha, \lambda) \equiv k(\alpha, 0, \lambda)=h\left(\alpha u_{0}+\tilde{u}(\alpha, \lambda), \lambda\right)$. Now,

(a) $D g(\alpha, \lambda) \cdot(\beta, \mu)=D h\left(\alpha u_{0}+\tilde{u}(\alpha, \lambda), \lambda\right) \cdot\left(\beta u_{0}+D \tilde{u}(\alpha, \lambda) \cdot(\beta, \mu), \mu\right)$,

(b) $D^{2} g(\alpha, \lambda) \cdot(\beta, \mu)^{2}=D^{2} h\left(\alpha u_{0}+\tilde{u}(\alpha, \lambda), \lambda\right) \cdot\left(\beta u_{0}+D \tilde{u}(\alpha, \lambda) \cdot(\beta, \mu), \mu\right)^{2}$

$$
+D h\left(\alpha u_{0}+\tilde{u}(\alpha, \lambda), \lambda\right) \cdot D^{2} \tilde{u}(\alpha, \lambda) \cdot(\beta, \mu)^{2},
$$

(c)

$$
\begin{aligned}
D^{3} g(\alpha, \lambda) \cdot(\beta, \mu)^{3}=D^{3} h\left(\alpha u_{0}+\tilde{u}(\alpha, \lambda), \lambda\right) \cdot\left(\beta u_{0}+D \tilde{u}(\alpha, \lambda) \cdot(\beta, \mu), \mu\right)^{3} \\
+3 D^{2} h\left(\alpha u_{0}+\tilde{u}(\alpha, \lambda), \lambda\right) \\
\cdot\left[\left(\beta u_{0}+D \tilde{u}(\alpha, \lambda) \cdot(\beta, \mu), \mu\right), D^{2} \tilde{u}(\alpha, \lambda) \cdot(\beta, \mu)^{2}\right] \\
+D h\left(\alpha u_{0}+\tilde{u}(\alpha, \lambda), \lambda\right) \cdot D^{3} \tilde{u}(\alpha, \lambda) \cdot(\beta, \mu)^{3} .
\end{aligned}
$$

Therefore

(i) $g(0,0)=h(0,0)=0$,

(ii) $D g(0,0)=0$ because $D h(0,0)=0$,

(iii) $D^{2} g(0,0)=0$ because

$$
D^{2} h(0,0) \cdot\left(\beta u_{0}, \mu\right)^{2}=\beta^{2} D^{2} h(0,0) \cdot\left(u_{0}, 0\right)^{2}=\beta^{2}\left\langle T u_{0}, u_{0}\right\rangle=\beta^{2}\left\langle 0, u_{0}\right\rangle=0,
$$

(iv) $D^{3} g(0,0) \cdot(\beta, \mu)^{3}=\beta^{3} \int_{\Omega} G^{\prime \prime \prime}(0) u_{0}^{3} d x+3 \mu \beta^{2} \int_{\Omega} u_{0}^{2} d x$

using the formula for $D^{3} h$; the terms involving $D^{2} h$ and $D h$ give 0 . we have

Assume (by normalizing) that $\int_{\Omega} u_{0}^{2} d x=1$ and assume $\int_{\Omega} u_{0}^{3} d x \neq 0$. Since $G^{\prime \prime \prime}(0) \neq 0$,

$$
g(\alpha, \lambda)=\frac{1}{3 !} D^{3} g(0,0) \cdot(\alpha, \lambda)^{3}+\cdots=k \alpha^{3}+\frac{1}{2} \lambda \alpha^{2}+\cdots, \quad k \neq 0 .
$$

Let us multiply $\alpha$ and $\lambda$ by constants to put this in the form

$$
g(\alpha, \lambda)=\alpha^{3}+3 \lambda \alpha^{2}+\cdots
$$

The higher order terms are divisible by $\alpha^{2}$, since $g(0, \lambda)=h(0, \lambda)=0$ (recall $\tilde{u}(0, \lambda)=0$ ), and $g_{\alpha}(0, \lambda)=0$ because $D h(0, \lambda)=0$. Let us put $g$ into normal form using the ideas in Wasserman [1975]. First note that $g(\alpha, \lambda)$ has the form

$$
g(\alpha, \lambda)=\alpha^{3} z(\alpha)+3 \alpha^{2} \lambda q(\alpha, \lambda)
$$


where $z(0)=1$ and $q(0,0)=1$. By the universal unfolding theorem for cubic singularities, there are functions $\beta(\alpha, \lambda), \sigma(\lambda)$ and $\tau(\lambda)$ such that

$$
g(\alpha, \lambda)=h(\beta(\alpha, \lambda), \sigma(\lambda), \tau(\lambda))
$$

where

$$
h(\beta, \sigma, \tau)=\beta^{3}+\sigma \beta+\tau
$$

and

$$
\beta(0,0)=0, \quad \beta_{\alpha}(0,0)>0, \quad \sigma(0)=0, \quad \tau(0)=0 .
$$

Using the chain rule in some straightforward calculations we find that

$$
\sigma^{\prime}(0)=0 \text { and } \sigma^{\prime \prime}(0)<0 .
$$

Thus, there is a further change of coordinates $\mu=\mu(\lambda)$ with $\mu(0)=0, \mu^{\prime}(0)>0$, such that

$$
g(\alpha, \lambda)=[\beta(\alpha, \lambda)]^{3}-3[\mu(\lambda)]^{2} \beta(\alpha, \lambda)+\tau(\lambda) .
$$

Since $g(0, \lambda)=0, \quad\left\{[\beta(0, \lambda)]^{2}-3[\mu(\lambda)]^{2}\right\} \beta(0, \lambda)+\tau(\lambda)=0 ;$ and since $g_{\alpha}(0, \lambda)=0$, $3\left\{[\beta(0, \lambda)]^{2}-[\mu(\lambda)]^{2}\right\} \beta_{\alpha}(0, \lambda)=0$. Since $\beta_{\alpha}(0, \lambda) \neq 0, \beta(0, \lambda)=\varepsilon \mu(\lambda)$ where $\varepsilon= \pm 1$. Hence $\tau(\lambda)=2 \varepsilon[\mu(\lambda)]^{3}$, so $g=\beta^{3}-3 \mu^{2} \beta+2 \varepsilon \mu^{3}$. Letting $\gamma=\beta-\varepsilon \mu$, we get $g=\gamma^{3}+$ $3 \varepsilon \gamma^{2} \mu$. If we differentiate each side of this equation twice with respect to $\alpha$ and once with respect to $\lambda$, and set $(\alpha, \lambda)=(0,0)$, we find that $1=\left[\gamma_{\alpha}(0,0)\right]^{2}\left[\gamma_{\lambda}(0,0)+\varepsilon \mu^{\prime}(0)\right]$. But $\gamma_{\lambda}(0,0)=\beta_{\lambda}(0,0)-\varepsilon \mu^{\prime}(0)=0$ and $\mu^{\prime}(0)>0$; therefore $\varepsilon=1$. Thus we obtain the normal form

$$
g(\alpha, \lambda)=\gamma^{3}+3 \mu \gamma^{2}
$$

in the new coordinates $(\gamma, \mu)$. Hence there is a change of coordinates respecting the parameter such that the higher order terms can be eliminated.

Note that $g$ is the potential function for a transcritical bifurcation: if we set $g_{\alpha}(\alpha, \lambda)=0$ we get

$$
3 \alpha^{2}+6 \lambda \alpha+2 \alpha(\cdots)=0
$$

The solution set is therefore the $\lambda$-axis and a curve tangent at $(0,0)$ to the line $\alpha+2 \lambda=0$. The expression $g(\alpha, \lambda)=\gamma^{3}+3 \mu \gamma^{2}$ puts the potential function for this bifurcation problem into normal form.

Normal forms for the equations $g_{\alpha}=0$ by coordinate changes respecting the parameter are found in Golubitsky and Schaeffer [1979]; see Marsden and Hughes [1983, Chap. 7] for simple proofs adequate for the present example. Golubitsky and Schaeffer point out that for many bifurcation problems, the equation $g_{\alpha}=0$ can be put into normal form by a coordinate change respecting the parameter, but the potential function $g$ cannot.

Our approach to Example 7 should be compared with, for example, Chillingworth [1974] and Zeeman [1976], which consider a one-dimensional problem in which difficulties with the function spaces do not occur (i.e the energy norm is a complete Hilbert space norm) and for which the bifurcation parameter is not treated as distinguished. The example of Beeson and Tromba [1981] has the function-space complications of our example (i.e. the energy norm is not complete) but has additional complications due to a group action. However there is no distinguished bifurcation parameter. 
Example 8 (E. N. Dancer). This example proves the conjecture in the introduction for Hilbert spaces. Let $H$ be a Hilbert space with inner product $\langle\cdot, \cdot\rangle$, and let $B$ : $H \times H \rightarrow \mathbb{R}$ be a continuous symmetric bilinear map. There is a bounded self-adjoint operator $L: H \rightarrow H$ such that $B(x, y)=\langle L x, y\rangle$. Suppose that $L$ is not an isomorphism. Let $f(x)=\frac{1}{2} B(x, x)$. We shall find a continuous homogeneous cubic polynomial $p$ : $H \rightarrow \mathbb{R}$ such that $f$ and $f+p$ are not $C^{1}$ right equivalent in any neighborhood of the origin. Thus any generalization of the Morse-Palais lemma in Hilbert space must place restrictions on the perturbation $p$.

Let $\sigma(L)$ denote the spectrum of $L$. Since $L$ is not an isomorphism, $0 \in \sigma(L)$.

Case 1. $N(L)=\varnothing$. Then $\nabla f(x)=L x \neq 0$ for $x \neq 0$. We shall find a continuous homogeneous cubic polynomial $p: H \rightarrow \mathbb{R}$ such that $\nabla(f+p)(x)=L x+\nabla p(x)=0$ at points $x$ arbitrarily close to 0 . Then $f$ and $f+p$ cannot be $C^{1}$ right equivalent.

There exist $w_{n} \in \sigma(L), w_{n} \neq 0$, such that $w_{n} \rightarrow 0$. For each $n$ let $I_{n}$ be a closed interval centered at $w_{n}$ such that the $I_{n}$ are disjoint and radius $\left(I_{n}\right)<\left|w_{n}\right| / 2$. Let $P_{n}$ be the orthogonal projection corresponding to $I_{n}$ that is given by the spectral theorem, and let $H_{n}=P_{n} H$. The subspaces $H_{n}$ are mutually orthogonal subspaces of $H$, invariant under $L$, and $L \mid H_{n}$ has spectrum lying in $I_{n}$.

Choose $e_{n} \in H_{n}$ such that $\left\|e_{n}\right\|=1$. Then $\left\|L e_{n}-w_{n} e_{n}\right\|<\left|w_{n}\right| / 2$. Let $z_{n}=L e_{n}-w_{n} e_{n}$. Decompose $z_{n}$ as $z_{n}=\sigma_{n} e_{n}+\tau_{n} y_{n}$ where $\left\langle e_{n}, y_{n}\right\rangle=0$ and $\left\|y_{n}\right\|=1$. If $z_{n}$ is a multiple of $e_{n}$, set $y_{n}=0, \tau_{n}=0$. Then $y_{n} \in H_{n}$ (by invariance of $H_{n}$ under $L$ ) and $\left|\sigma_{n}\right|,\left|\tau_{n}\right|<\left|w_{n}\right| / 2$. We conclude that $L e_{n}=\mu_{n} e_{n}+\tau_{n} y_{n}$ where $\mu_{n}=w_{n}+\sigma_{n}$. Thus $\left|w_{n}\right| / 2<\left|\mu_{n}\right|<3\left|w_{n}\right| / 2$.

Define $p_{n}$ on $\operatorname{span}\left\{e_{n}, y_{n}\right\}$ by $p_{n}\left(\alpha e_{n}+\beta y_{n}\right)=\alpha^{3}+\left(3 \tau_{n} / \mu_{n}\right) \alpha^{2} \beta$. Notice that $\left|3 \tau_{n} / \mu_{n}\right|$ $<\left(3\left|w_{n}\right| / 2\right) /\left(\left|w_{n}\right| / 2\right)=3$. We find that $L\left(\gamma_{n} e_{n}\right)+\nabla p_{n}\left(\gamma_{n} e_{n}\right)=0$ provided

$$
3 \gamma_{n}^{2}+\gamma_{n} \mu_{n}=0
$$

and

$$
\frac{3 \tau_{n}}{\mu_{n}} \gamma_{n}^{2}+\gamma_{n} \tau_{n}=0
$$

i.e., provided $\gamma_{n}=-\mu_{n} / 3$.

Finally, define $p$ on $H$ by $p=\Sigma p_{n}$. Since all the $e_{n}$ 's and $y_{n}$ 's are mutually orthogonal, $p$ is a continuous cubic polynomial. by

(Proof. $p(x)=T(x, x, x)$ where $T(u, v, w)$ is the symmetric trilinear map defined

$$
\begin{aligned}
T(u, v, w)=\sum & \left\langle u, e_{n}\right\rangle\left\langle v, e_{n}\right\rangle\left\langle w, e_{n}\right\rangle+\sum \frac{\tau_{n}}{\mu_{n}}\left\langle u, y_{n}\right\rangle\left\langle v, e_{n}\right\rangle\left\langle w, e_{n}\right\rangle \\
& +\sum \frac{\tau_{n}}{\mu_{n}}\left\langle u, e_{n}\right\rangle\left\langle v, y_{n}\right\rangle\left\langle w, e_{n}\right\rangle+\sum \frac{\tau_{n}}{\mu_{n}}\left\langle u, e_{n}\right\rangle\left\langle v, e_{n}\right\rangle\left\langle w, y_{n}\right\rangle .
\end{aligned}
$$

Each of these four sums is a bounded trilinear map. For example, the second sum is estimated as follows:

$$
\begin{aligned}
\left|\sum \frac{\tau_{n}}{\mu_{n}}\left\langle u, y_{n}\right\rangle\left\langle v, e_{n}\right\rangle\left\langle w, e_{n}\right\rangle\right|<\sum\left|\left\langle u, y_{n}\right\rangle\left\langle v, e_{n}\right\rangle\left\langle w, e_{n}\right\rangle\right| \\
\leq\left[\sum\left\langle u, y_{n}\right\rangle^{2}\right]^{1 / 2}\left[\sum\left\langle v, e_{n}\right\rangle^{2}\left\langle w, e_{n}\right\rangle^{2}\right]^{1 / 2} \\
\left.\leq\left[\sum\left\langle u, y_{n}\right\rangle^{2}\right]^{1 / 2} \cdot\left[\sum\left\langle v, e_{n}\right\rangle^{2} \cdot \sum\left\langle w, e_{n}\right\rangle^{2}\right]^{1 / 2} \leq\|u\|\|v\|\|w\| . \quad\right)
\end{aligned}
$$

We have $L\left(\gamma_{n} e_{n}\right)+\nabla p\left(\gamma_{n} e_{n}\right)=0$ where $\gamma_{n} \rightarrow 0$. 
Case 2. $N(L) \neq \varnothing$. Let $\left\{e_{n}\right\}$ be an orthonormal basis for $N(L)$. Let $p(x)=$ $\Sigma\left\langle x, e_{n}\right\rangle^{3}$. Then $\nabla f(x)=0$ for all $x \in N(L)$, but $\nabla(f+p)(x) \neq 0$ if $x \neq 0$. Thus $f$ and $f+p$ are not $C^{1}$ right equivalent.

\section{REFERENCES}

M. BeESON AND A. J. TROMBA [1981], The cusp catastrophe of Thom in the bifurcation of minimal surfaces, preprint \#462 SFB, Bonn, Manus. Math., to appear.

D. R. J. ChIllingworth [1974], The catastrophe of a buckling beam, Lecture Notes in Mathematics 468, Springer-Verlag, New York, pp. 86-91.

D. R. J. Chillingworth, J. E. MARSDEN AND Y. H. WAN [1982], Symmetry and bifurcation in three-dimensional elasticity, Arch. Rat. Mech. Anal., 80, pp. 295-331.

Y. Choquet-Bruhat, A. Fischer AND J. Marsden [1979], Maximal hypersurfaces and positivity of mass, in Isolated Gravitating Systems in General Relativity, J. Ehlers, ed., Proc. 1976 Varena conference, Italian Physical Society, pp. 322-395.

A. Friedman [1969], Partial Differential Equations, Holt, Rinehart, and Winston, New York.

M. GOlubitsky AND J. MARSDEN [1983], The Morse lemma in infinite dimensions via singularity theory, this Journal, this issue, pp. 1037-1044.

M. Golubitsky AND D. SCHAEFFer [1979], A theory for imperfect bifurcation via singularity theory, Comm. Pure. Appl. Math., 32, pp. 21-98.

J. MARSDEN [1968], Hamiltonian one parameter groups, Arch. Rat. Mech. Anal., 28, pp. 323-361.

J. MARSDEN AND T. Hughes [1983], Mathematical Foundations of Elasticity, Prentice-Hall, Englewood Cliffs, NJ.

J. Milnor [1963], Morse Theory, Princeton Univ. Press, Princeton, NJ.

R. PALAIs [1963], Morse theory on Hilbert manifolds, Topology 2, pp. 299-340. [1968], Foundations of Global Non-linear Analysis, Benjamin, Menlo Park, CA. [1969], The Morse lemma on Banach spaces, Bull. Amer. Math. Soc., 75, pp. 968-971.

A. J. Tromba [1976], Almost Riemannian structures on Banach manifolds, the Morse lemma and the Darboux theorem, Canad. J. Math., 28, pp. 640-652. [1981], A sufficient condition for a critical point of a functional to be a minimum and its application to Plateau's problem, preprint \#408, SFB Bonn, Math. Ann., to appear.

G. WASSERMAN [1975], Stability of unfoldings in space and time, Acta Math., 135, pp. 57-128.

C. ZeEman [1976], Euler buckling, Lecture Notes in Mathematics 525, Springer-Verlag, New York, pp. 373-395. 